\title{
Research on Resonance Mechanism and Collaborative Optimization of Double-cavity Self- Excited Oscillating Pulse Cavitation Jet Nozzle
}

xiaoming yuan ( $\nabla$ xiaomingbingbing@163.com )

Yanshan University https://orcid.org/0000-0001-5779-1366

\section{Li Wang}

Yanshan University

\section{Weidong Wang}

Yanshan University

\section{Lijie Zhang}

Yanshan University

\section{Yong Zhu}

Jiangsu University

\section{Original Article}

Keywords: Self excited oscillation, Pulsed cavitation jet, The nozzle, Resonance mechanism, Collaborative optimization

Posted Date: June 10th, 2021

DOI: https://doi.org/10.21203/rs.3.rs-593170/v1

License: (1) This work is licensed under a Creative Commons Attribution 4.0 International License. Read Full License 


\section{Abstract}

The peak value and pulsation amplitude of the self-excited oscillating pulse cavitation jet nozzle are important indexes to evaluate the jet performance. It is of great significance in theory and engineering practice to predict the peak value of the self-excited oscillating pulse cavitation jet nozzle accurately. In order to investigate the evolution mechanism of the inner and outer flow field of a double-cavity selfexcited oscillation pulse cavitation jet nozzle, a simulation model of the jet impact test of the nozzle was established. Before entrance rounded corners, former cavity cavity diameter, cavity cavity length, before the cavity under the nozzle diameter, cavity, the cavity cavity after entry the rounded, lumen diameter, cavity length and cavity after cavity under the nozzle diameter as design variables, and strike force to combat force peak pulse amplitude as the target variable, the orthogonal experiment method, back propagation neural network combined with non dominated sorting genetic algorithm, The collaborative optimization design method of self-excited oscillating pulse cavitation jet nozzle was determined. Based on the collaborative optimization results, the 3D printing technology was used to manufacture the visualization test model of the flow field of the self-excited oscillating pulse cavitation jet nozzle, and the experimental verification was carried out. The results show that when the inlet pressure is $2 \mathrm{MPa}$, the main and secondary order of the influences of various factors on the jet performance of the nozzle is the nozzle diameter under the front cavity, the diameter of the back cavity, the diameter of the front cavity, the length of the front cavity, the nozzle diameter under the back cavity, the cavity distance, the fillet of the back cavity, the fillet of the front cavity and the length of the back cavity. Compared with the optimal result of orthogonal test, the amplitude of impact pulsation and the peak value of impact force are increased by $14.61 \%$ and $2.42 \%$ respectively. The optimal structure of the nozzle determined by collaborative optimization can produce obvious pulse cavitation jet, and the cavitation region of the nozzle cavity contracts periodically with time. The higher the inlet pressure, the higher the cavitation intensity and the higher the content of hollow bubble. This study can promote the development of jet performance calculation of self-excited oscillation pulse cavitation jet nozzles, and provide support for the design of self-excited oscillation pulse cavitation jet nozzles.

\section{Full-text}

Due to technical limitations, full-text HTML conversion of this manuscript could not be completed. However, the manuscript can be downloaded and accessed as a PDF.

\section{Figures}




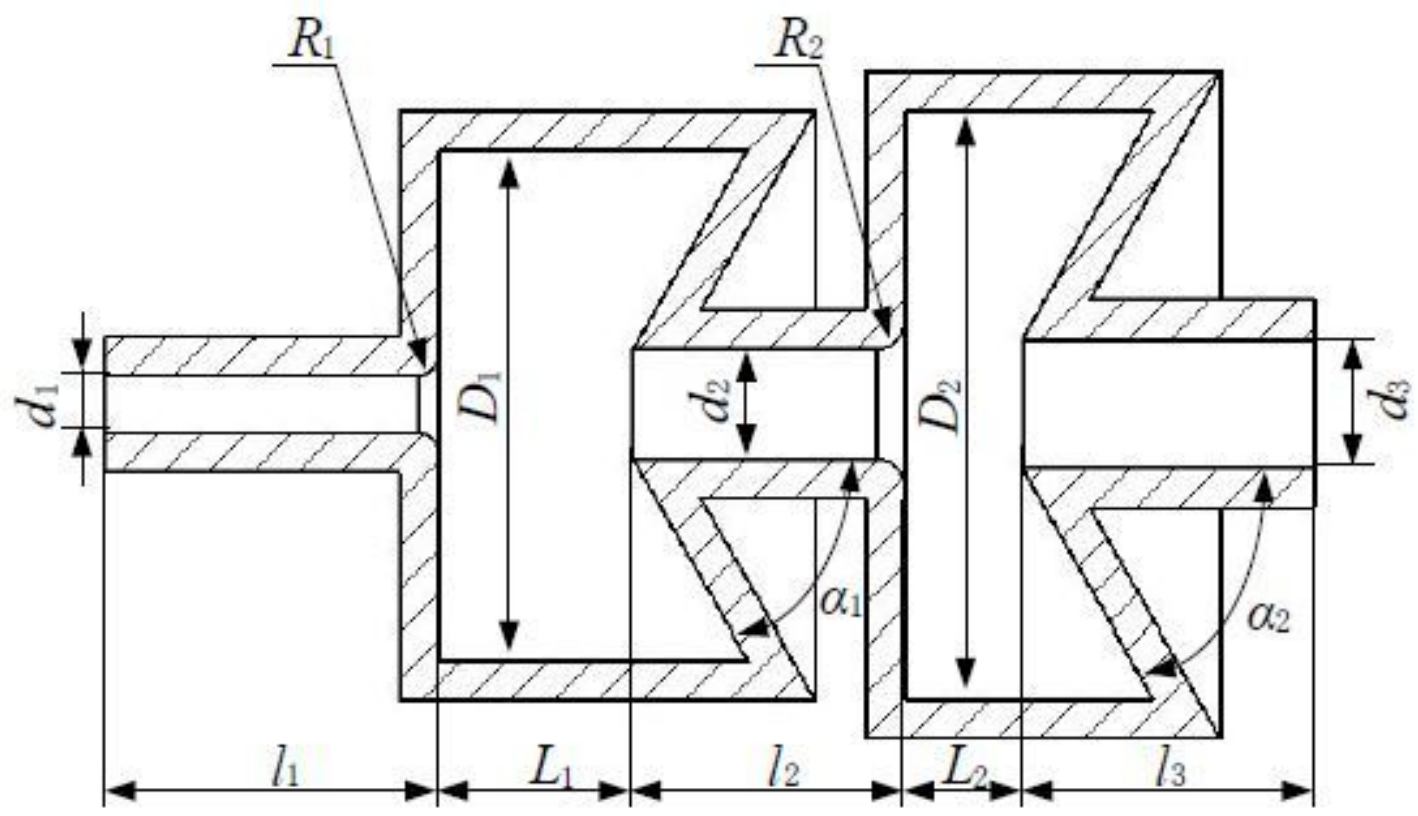

Figure 1

Nozzle geometry model

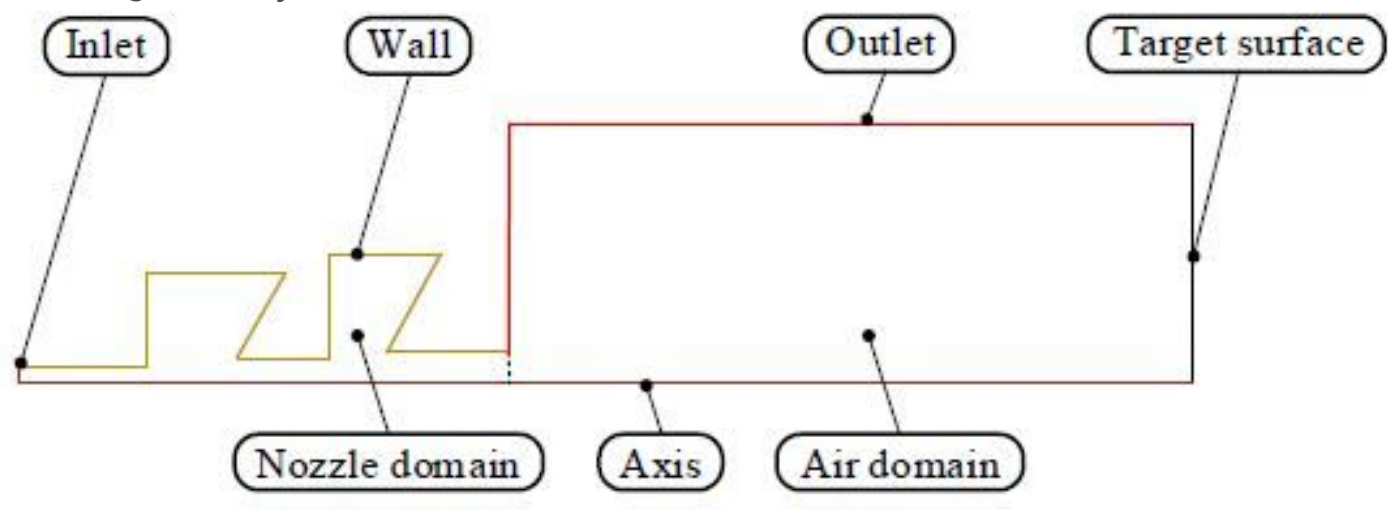

Figure 2

Nozzle simulation calculation domain model 


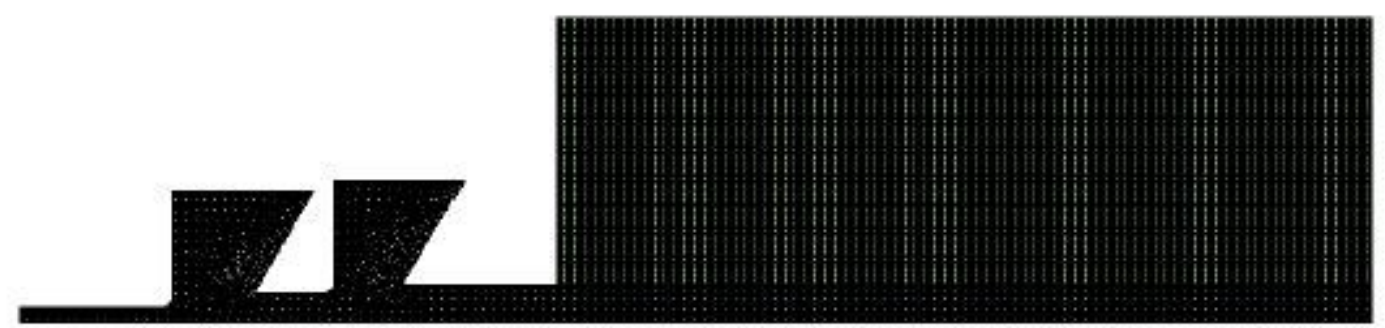

(a)

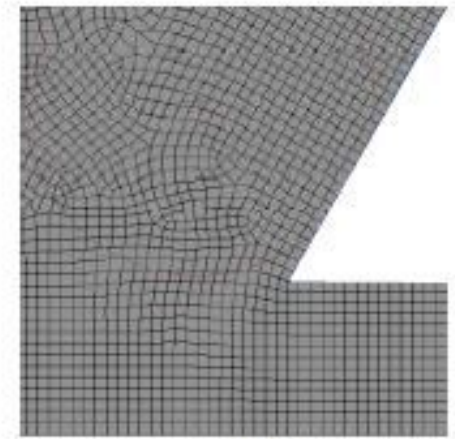

(b)

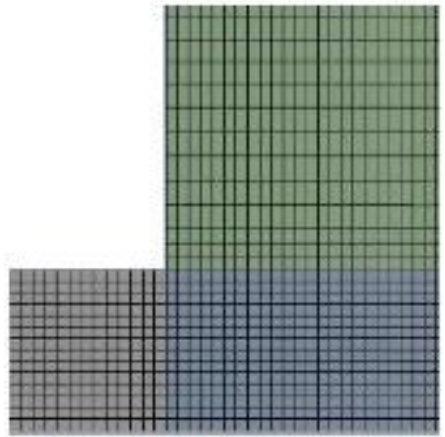

(c)

Figure 3

The overall and local mesh models of nozzle field were calculated: (a) The nozzle as a whole; (b) Cavity position; (c) Exit position

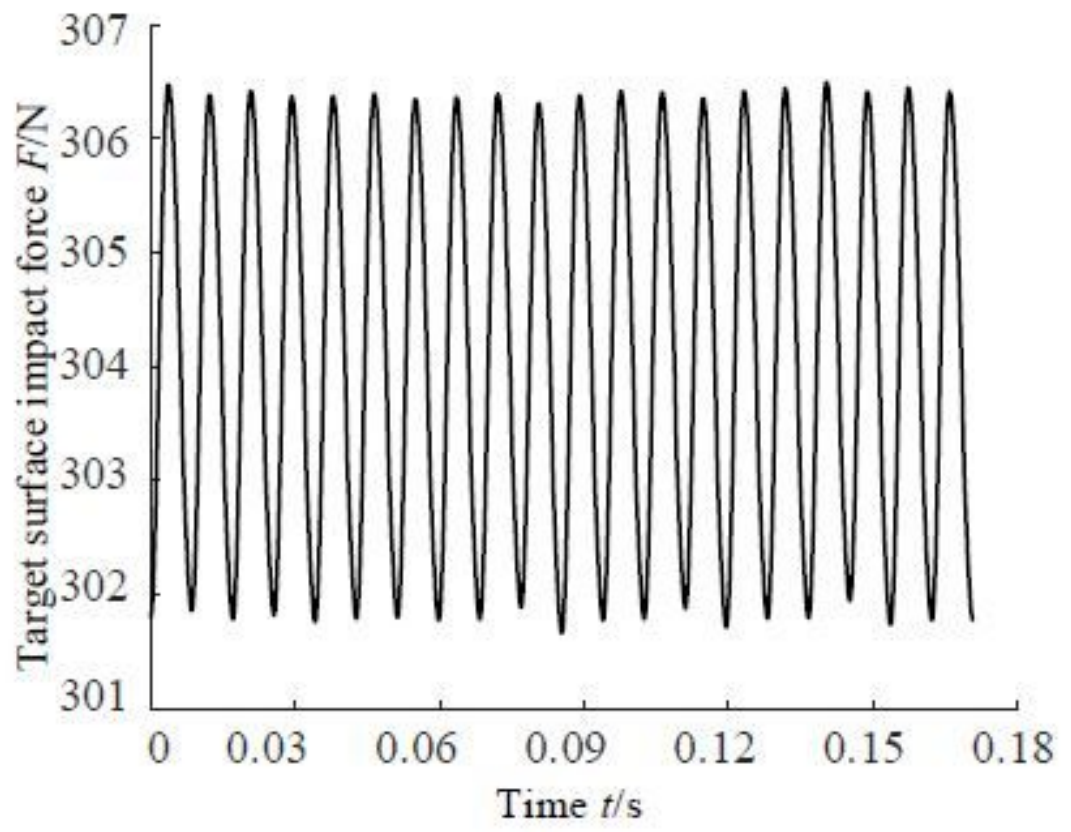

Figure 4

Test 2. Hit force curve of target surface 


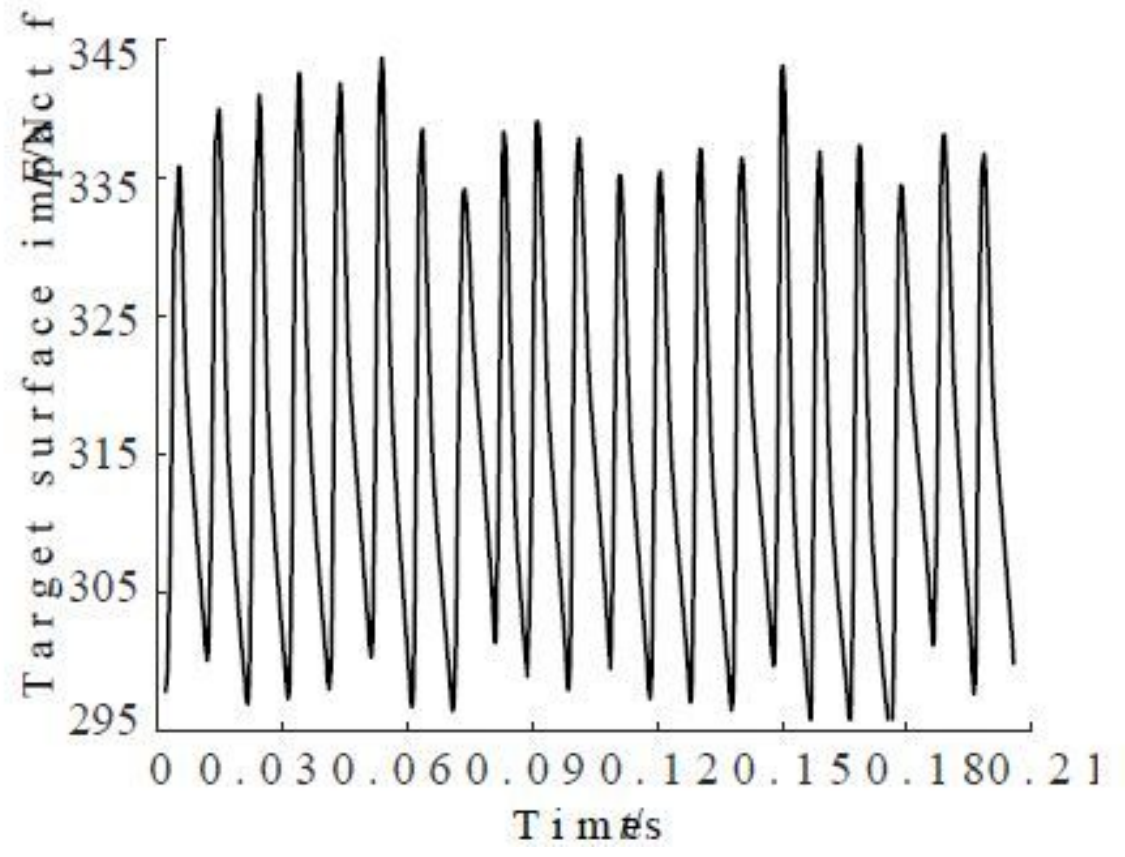

Figure 5

Test 7. Hit force curve of target surface

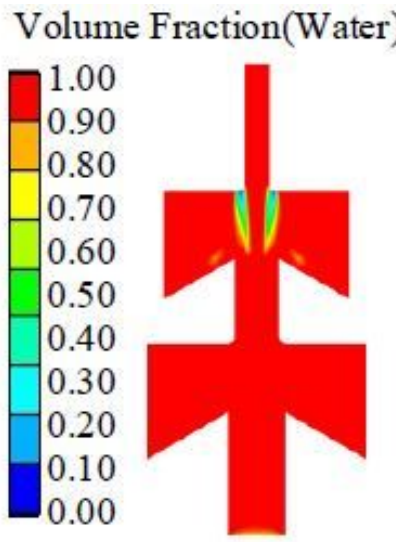

(a)

Volume Fraction(Water)

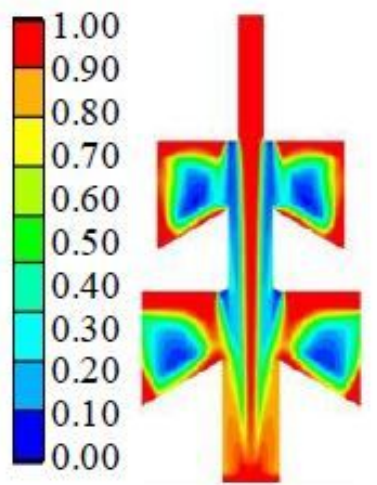

(e)

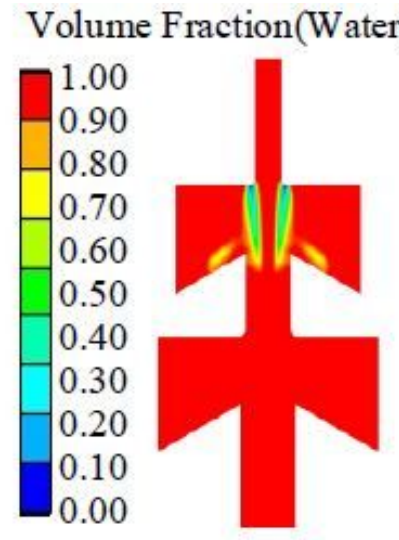

(b)

Volume Fraction(Water)

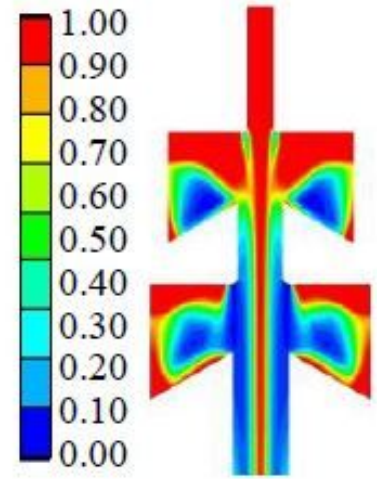

(f)

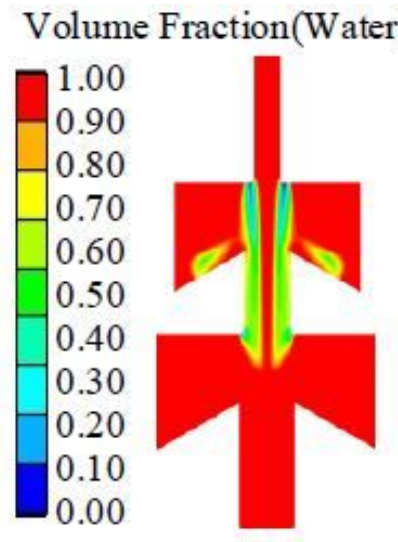

(c)

Volume Fraction(Water)

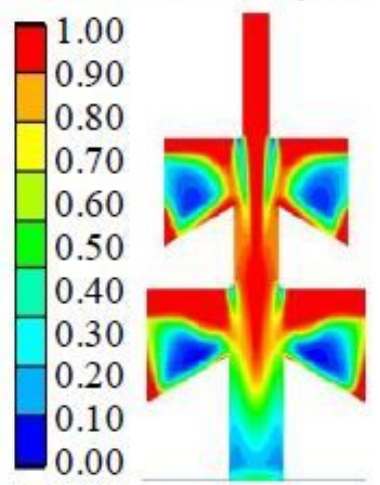

(g)

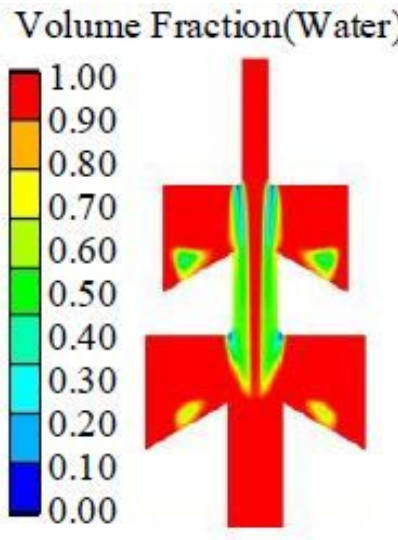

(d)

Volume Fraction(Water)

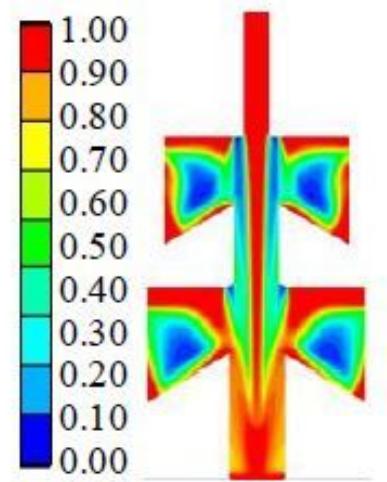

(h)

Figure 6 
Experiment 2 Evolution process of cavitation vortex: (a) $t=0.0027 \mathrm{~s}$; (b) $t=0.003 \mathrm{~s}$; (c) $t=0.004 \mathrm{~s}$; (d) $t=0.005 s ;(e) t=0.120 s ;$ (f) $t=0.124 s ;$ (g) $t=0.128 s ;(h) t=0.130 s$

Volume Fraction(Water)

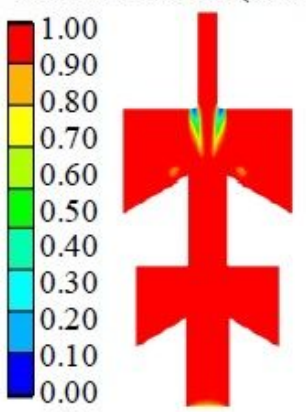

(a)

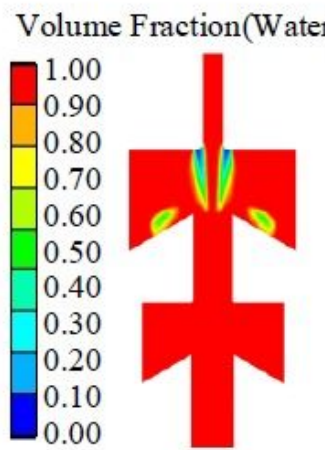

(b)

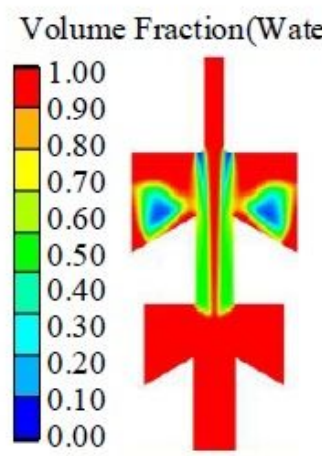

(c)

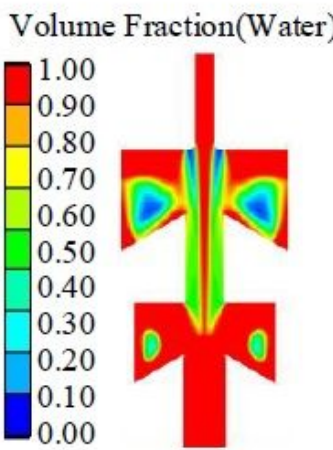

(d)

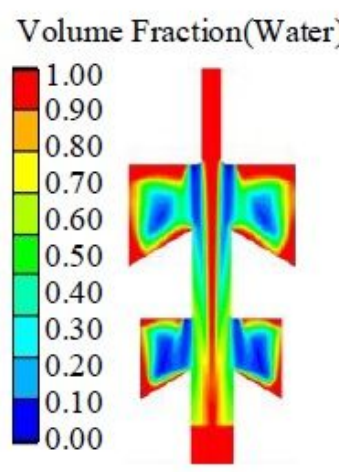

(e)

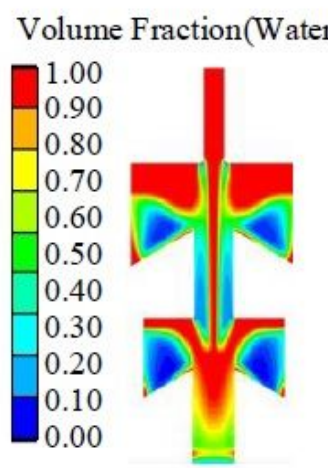

(f)

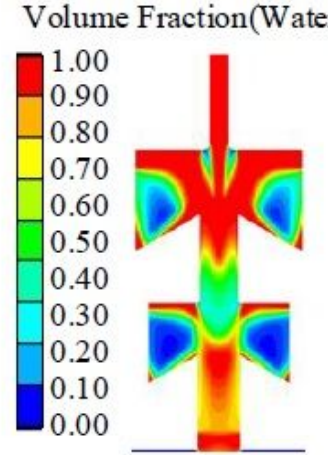

(g)

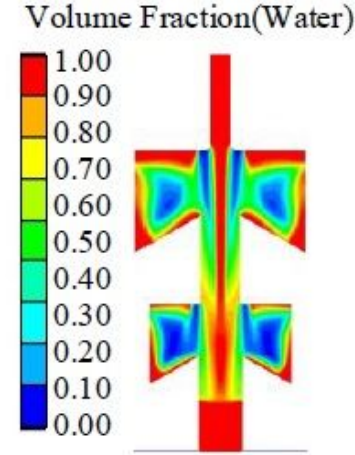

(h)

\section{Figure 7}

Experiment 7 Evolution process of cavitation vortex: (a) $t=0.002 \mathrm{~s}$; (b) $t=0.003 \mathrm{~s}$; (c) $t=0.01 \mathrm{~s}$; (d) $t=0.011 \mathrm{~s}$; (e) $t=0.151 \mathrm{~s}$; (f) $t=0.154 \mathrm{~s} ;$ (g) $t=0.156 \mathrm{~s}$; (h) $t=0.160 \mathrm{~s}$ 


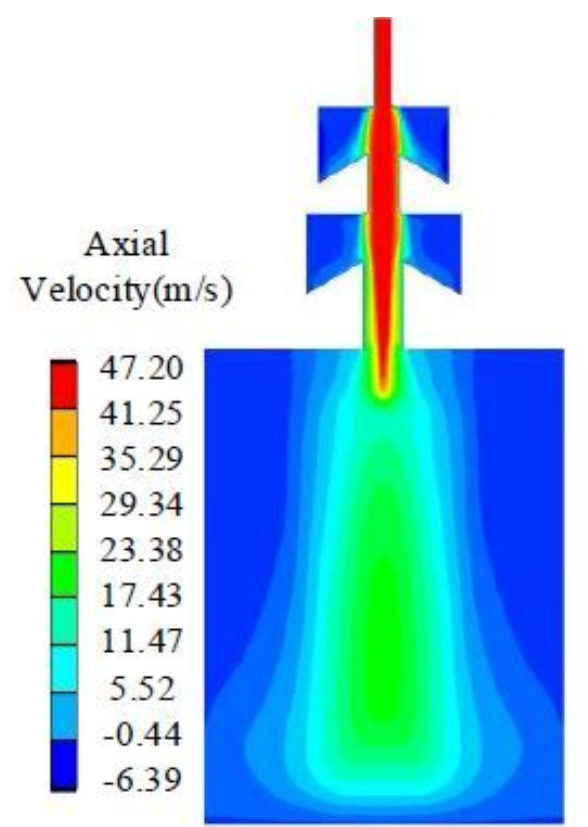

(a)

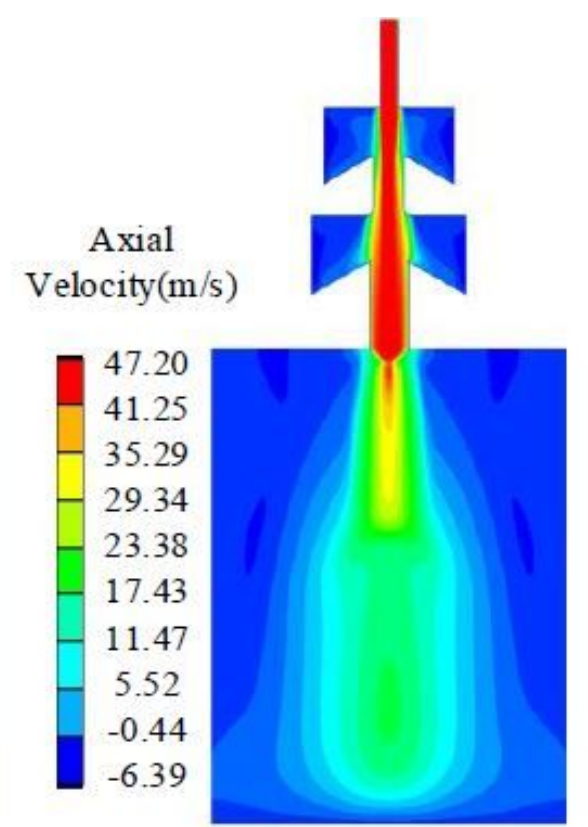

(b)

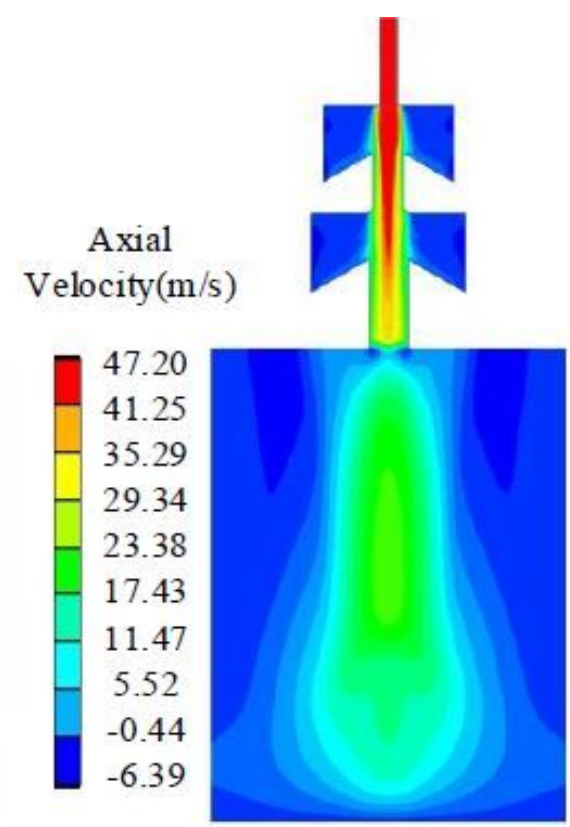

(c)

\section{Figure 8}

Experiment 2 Cloud map of axial velocity distribution of flow field at different moments: (a) $t=0.120 \mathrm{~s}$; (b) $t=0.124 s ;(c) t=0.128 s$

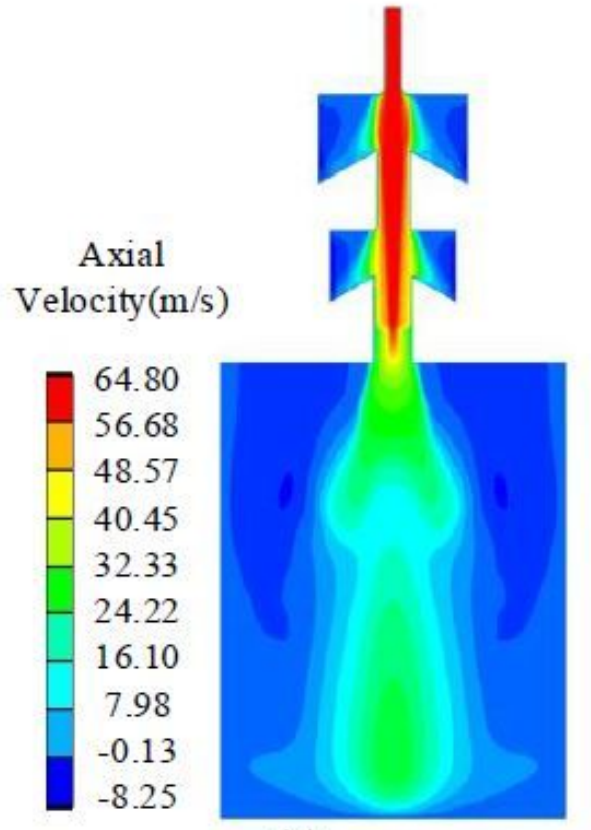

(a)

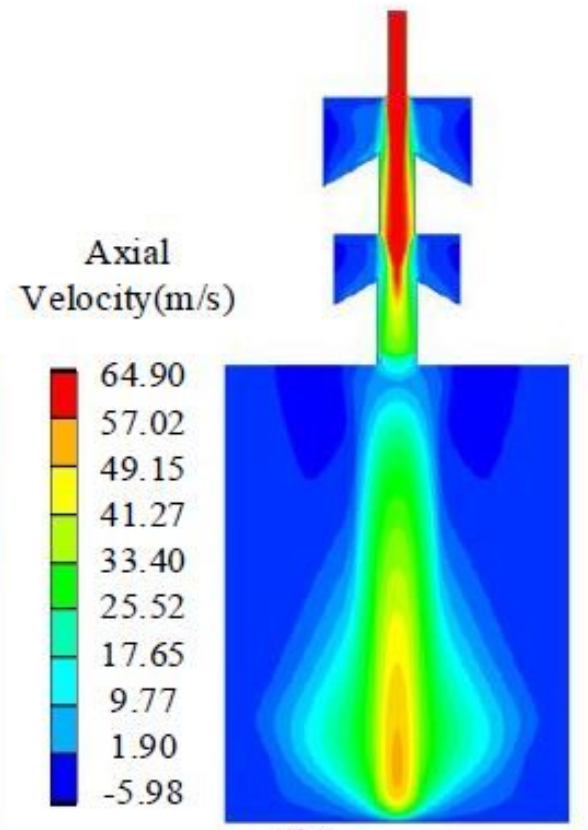

(b)

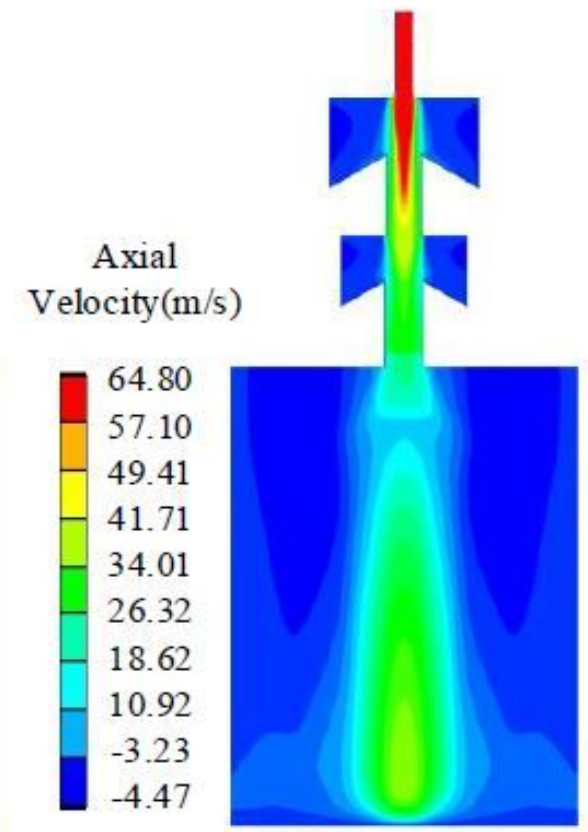

(c)

Figure 9

Experiment 7 Cloud map of axial velocity distribution of flow field at different moments: (a) $t=0.151 \mathrm{~s}$; (b) $t=0.154 \mathrm{~s} ;$ (c) $t=0.156 \mathrm{~s}$ 


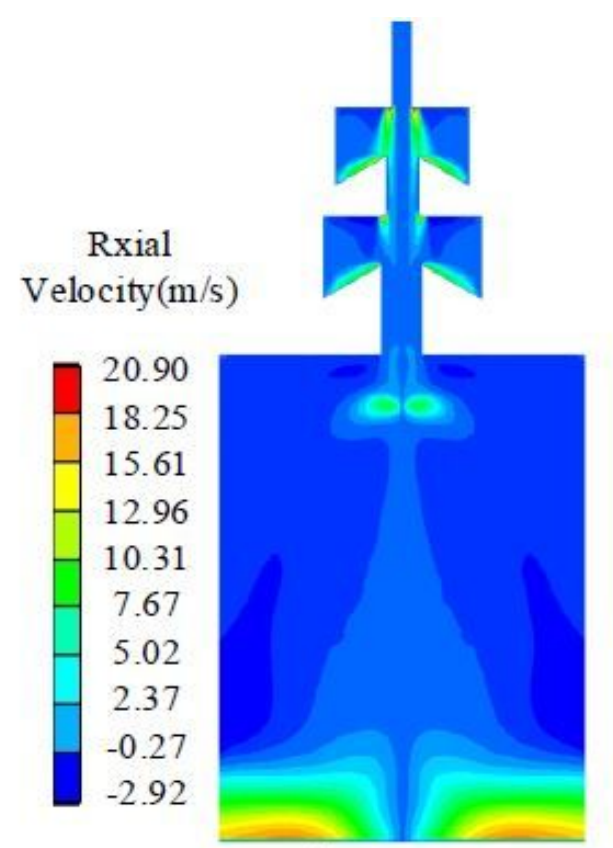

(a)

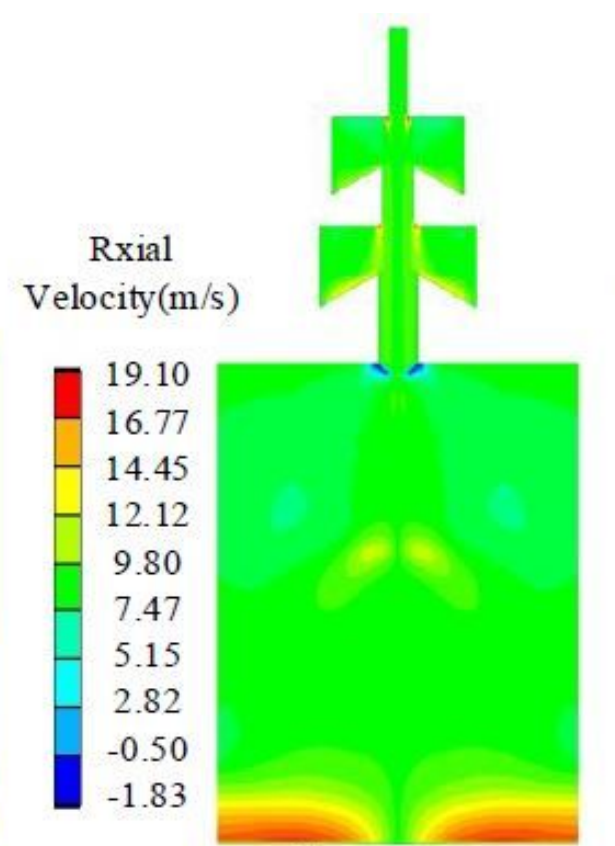

(b)

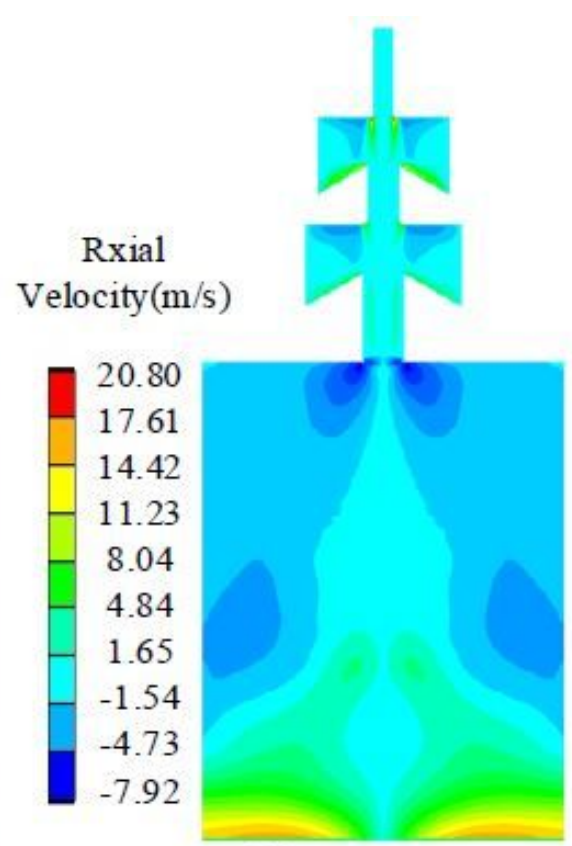

(c)

\section{Figure 10}

Experiment 2 Radial velocity distribution cloud image of flow field at different moments: (a) $t=0.120 \mathrm{~s}$; (b) $\mathrm{t}=0.124 \mathrm{~s} ;$ (c) $\mathrm{t}=0.128 \mathrm{~s}$

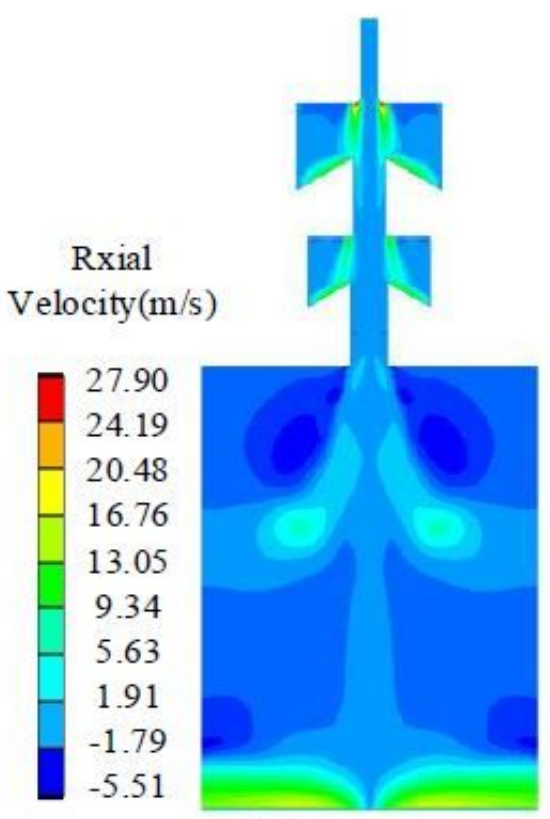

(a)

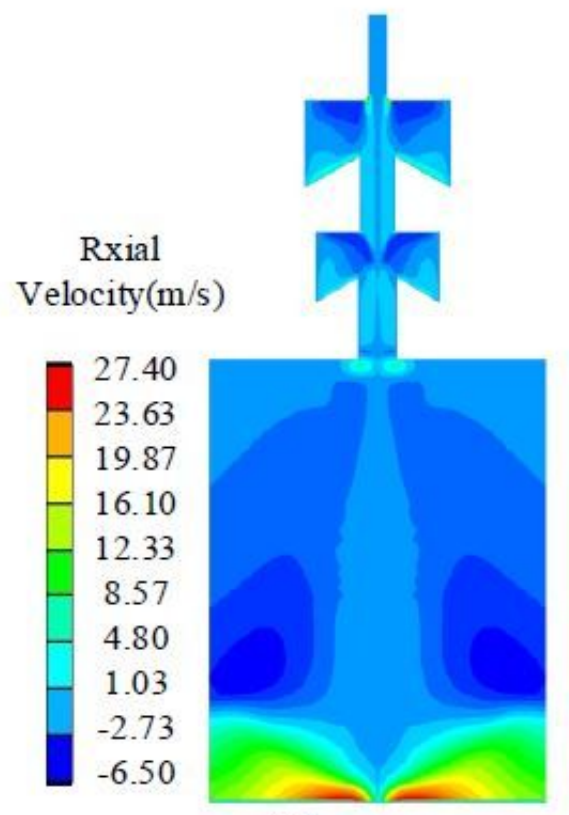

(b)

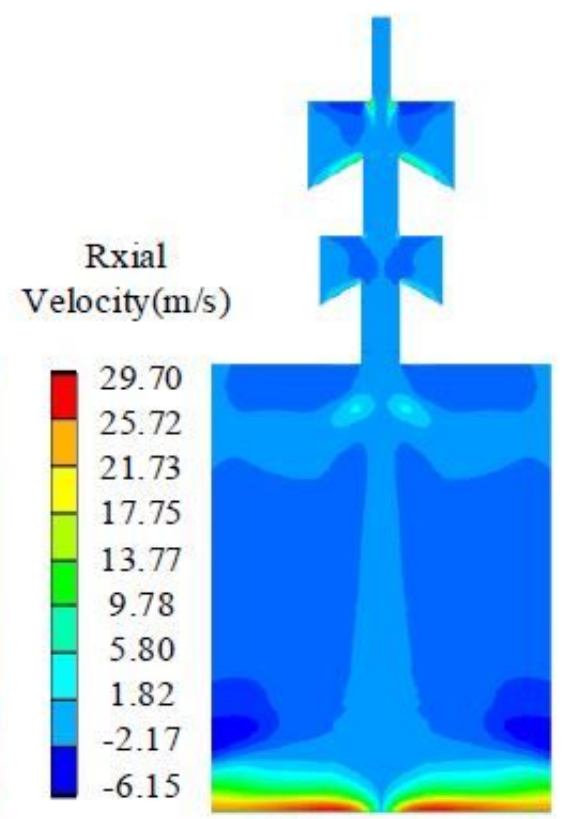

(c)

\section{Figure 11}

Experiment 7 Radial velocity distribution cloud map of flow field at different moments: (a) $t=0.151 \mathrm{~s}$; (b) $t=0.154 \mathrm{~s} ;(\mathrm{c}) \mathrm{t}=0.156 \mathrm{~s}$ 


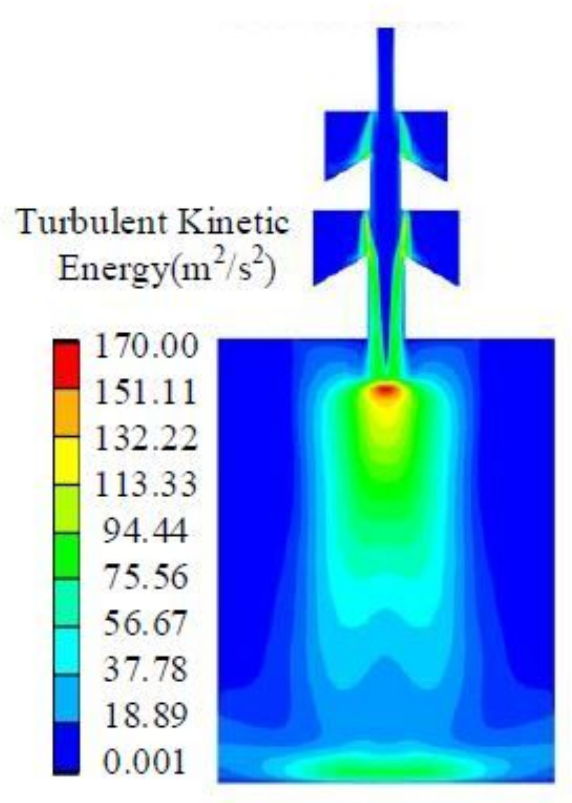

(a)

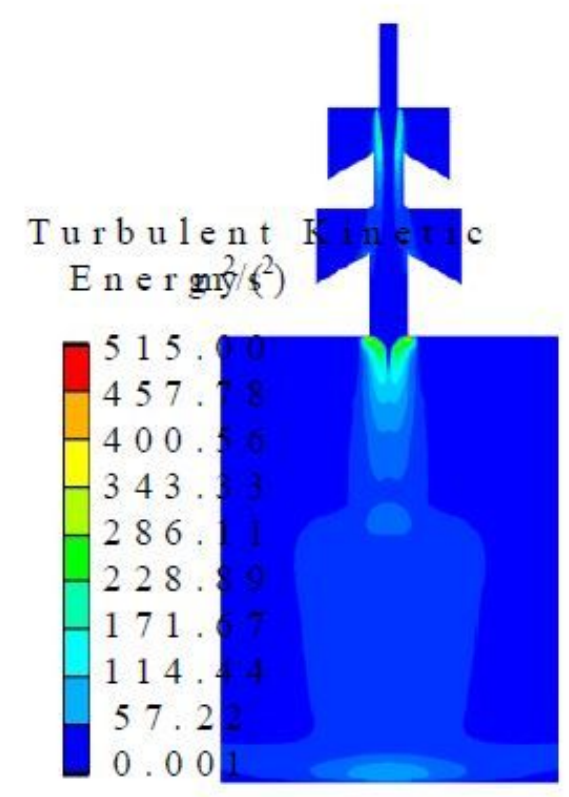

(b)

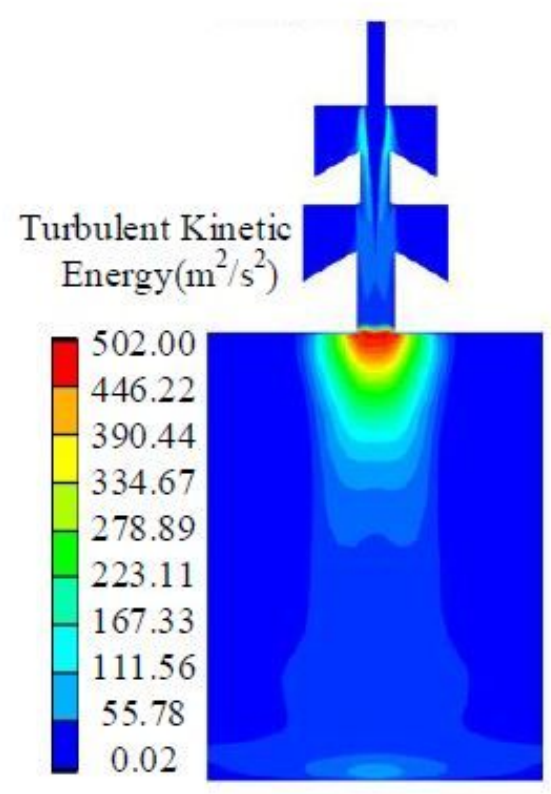

(c)

Figure 12

Experiment 2 Cloud map of turbulent kinetic energy distribution in the flow field at different moments: (a) $t=0.120 s ;(b) t=0.124 s ;(c) t=0.128 s$

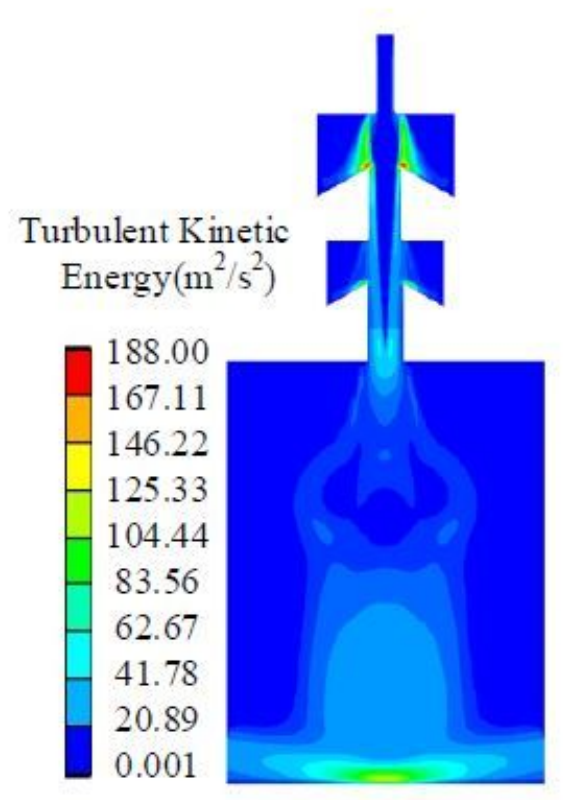

(a)

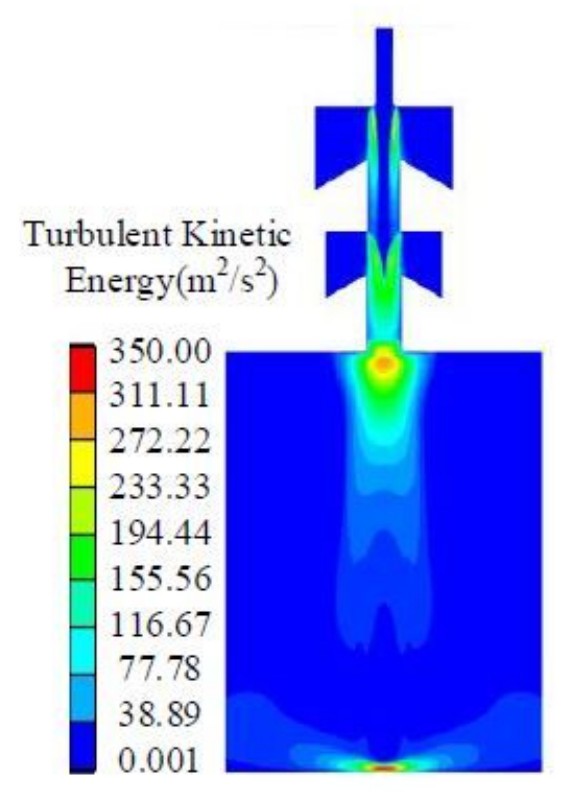

(b)

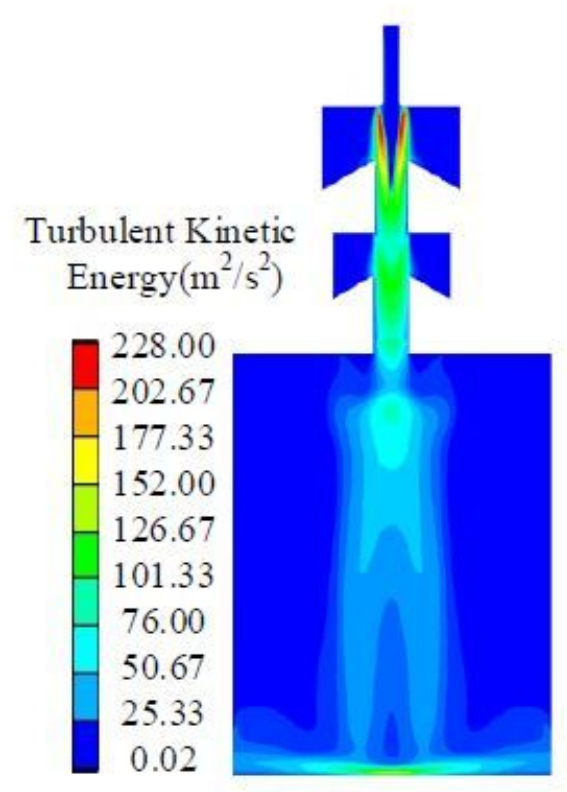

(c)

\section{Figure 13}

Experiment 7 Cloud map of turbulent kinetic energy distribution in the flow field at different moments: (a) $t=0.151 \mathrm{~s} ;$ (b) $t=0.154 \mathrm{~s} ;$ (c) $t=0.156 \mathrm{~s}$ 


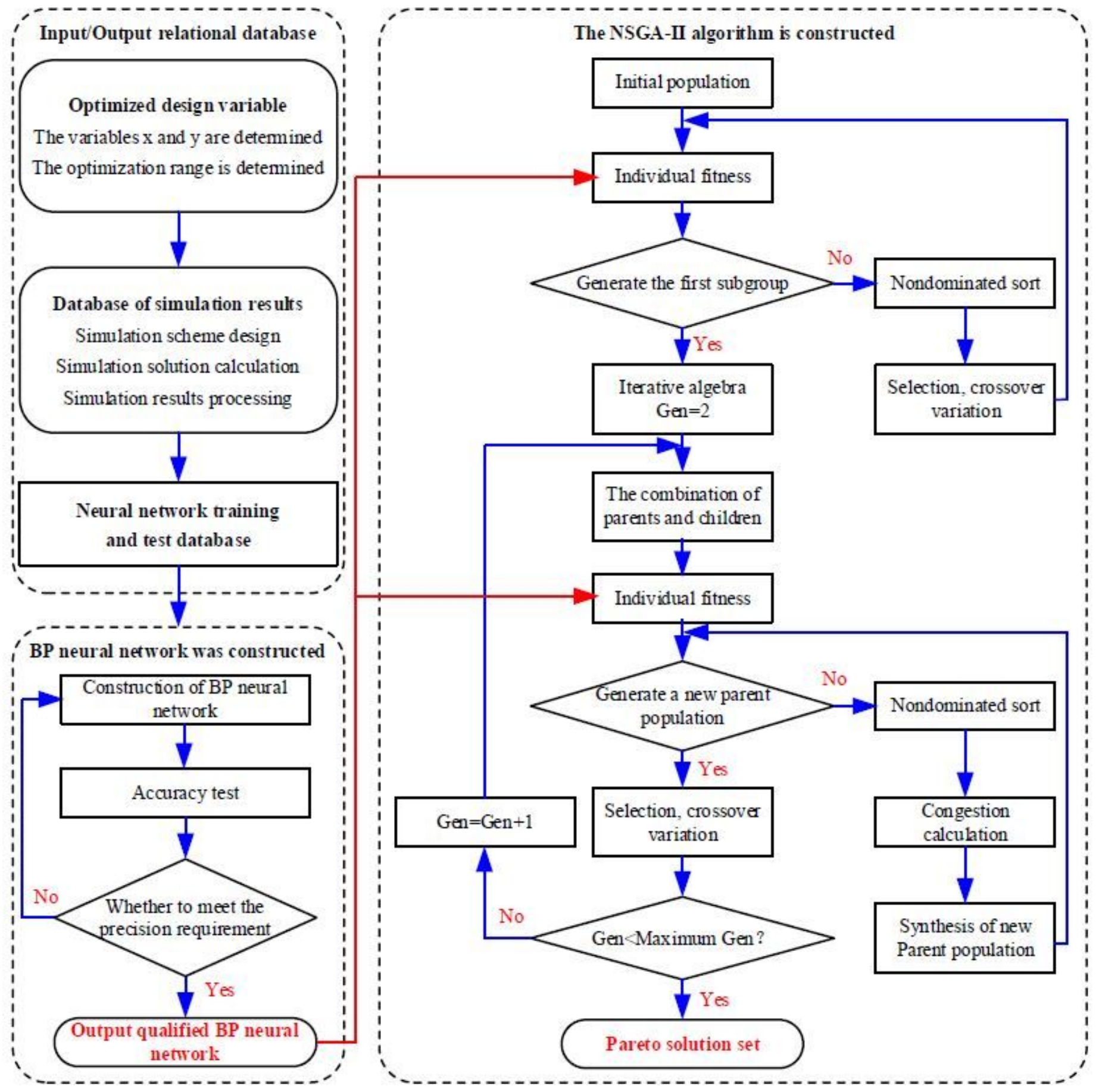

Figure 14

Flow chart of collaborative optimization design scheme 


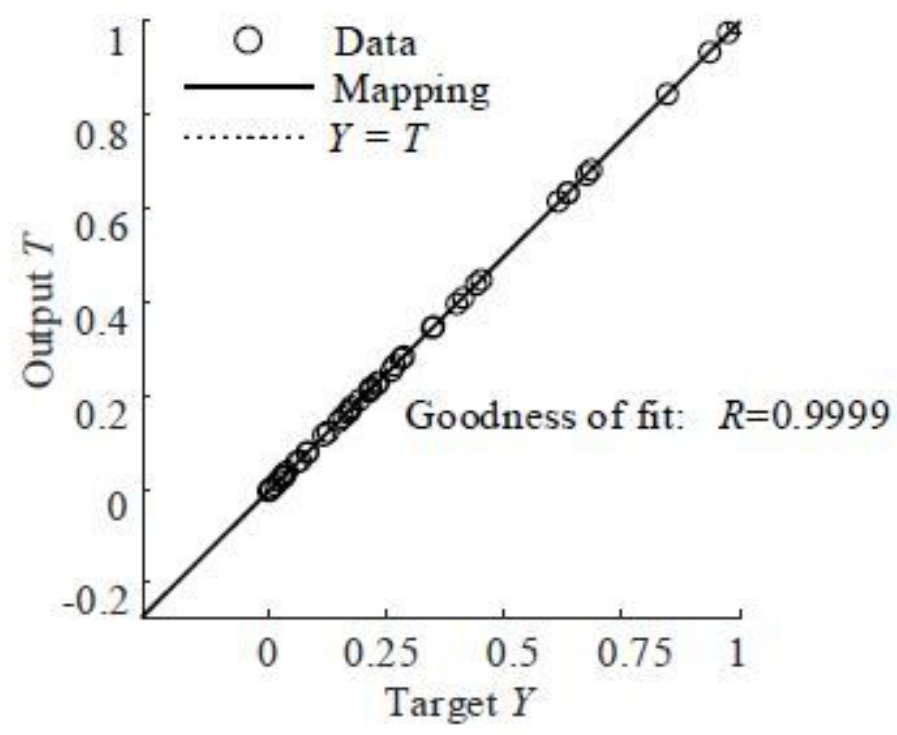

Figure 15

Test results of BP neural network in collaborative optimization

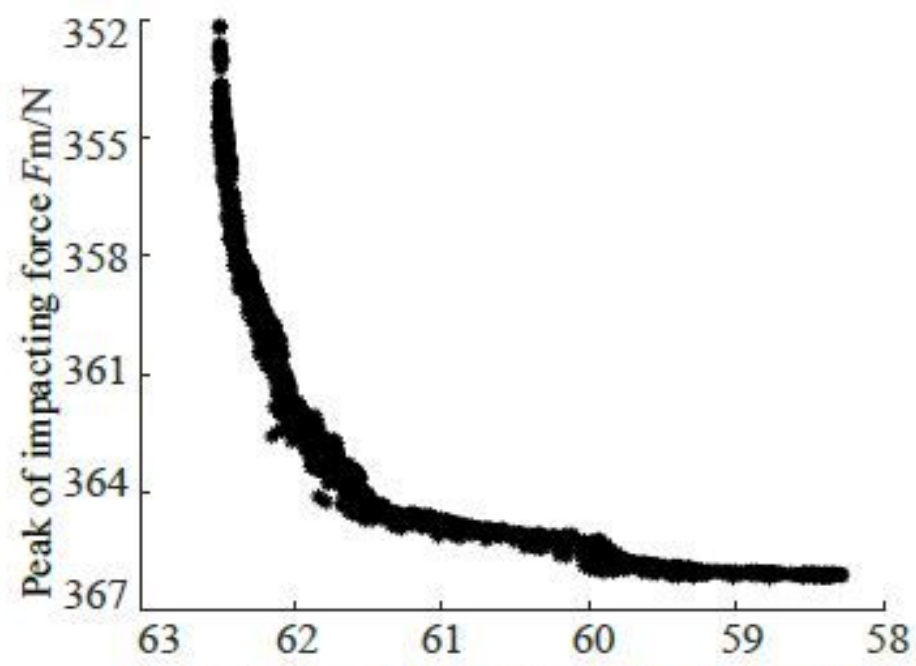

Pulsation amplitude of impacting force $\Delta F / \mathrm{N}$

Figure 16

Pareto optimal solution set under nozzle co-optimization 


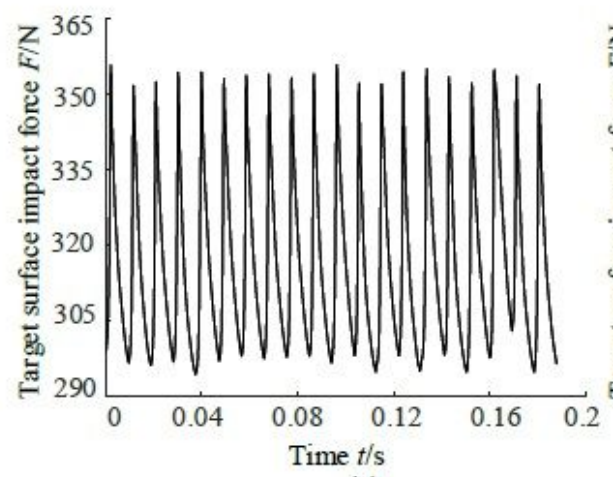

(a)

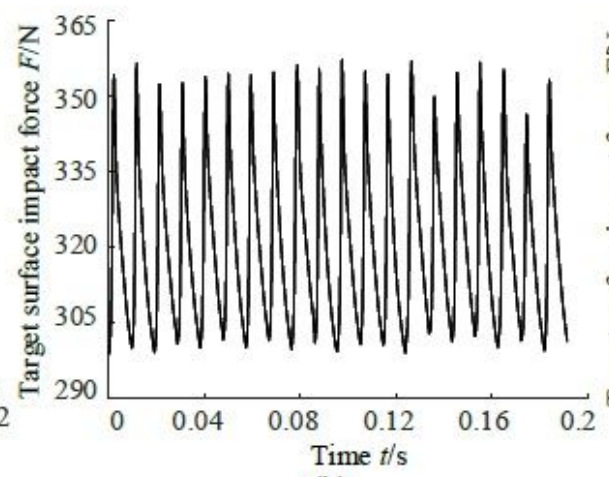

(b)

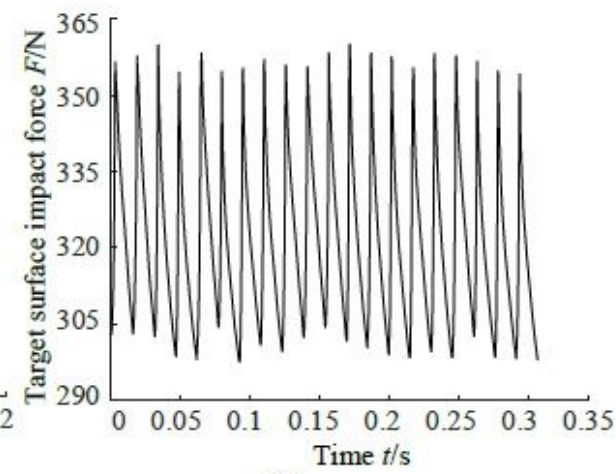

(c)

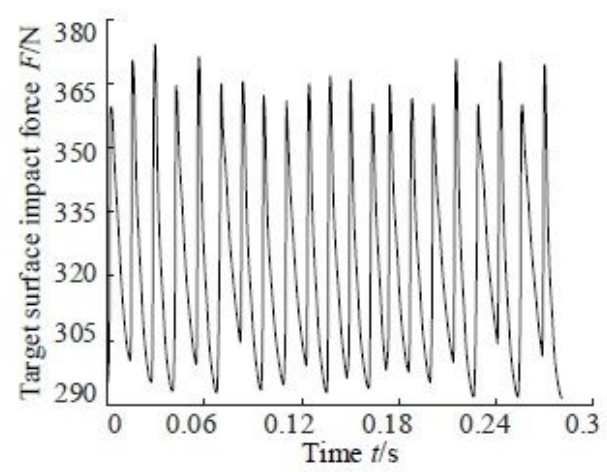

(d)

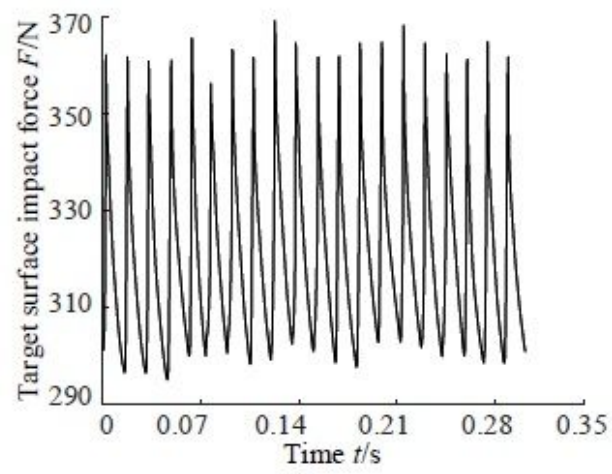

(e)

\section{Figure 17}

The striking force waveform of target surface under different nozzle structures: (a) Test 1; (b) Test 2; (c) Test 3; (d) Test 4; (e) Test 5 


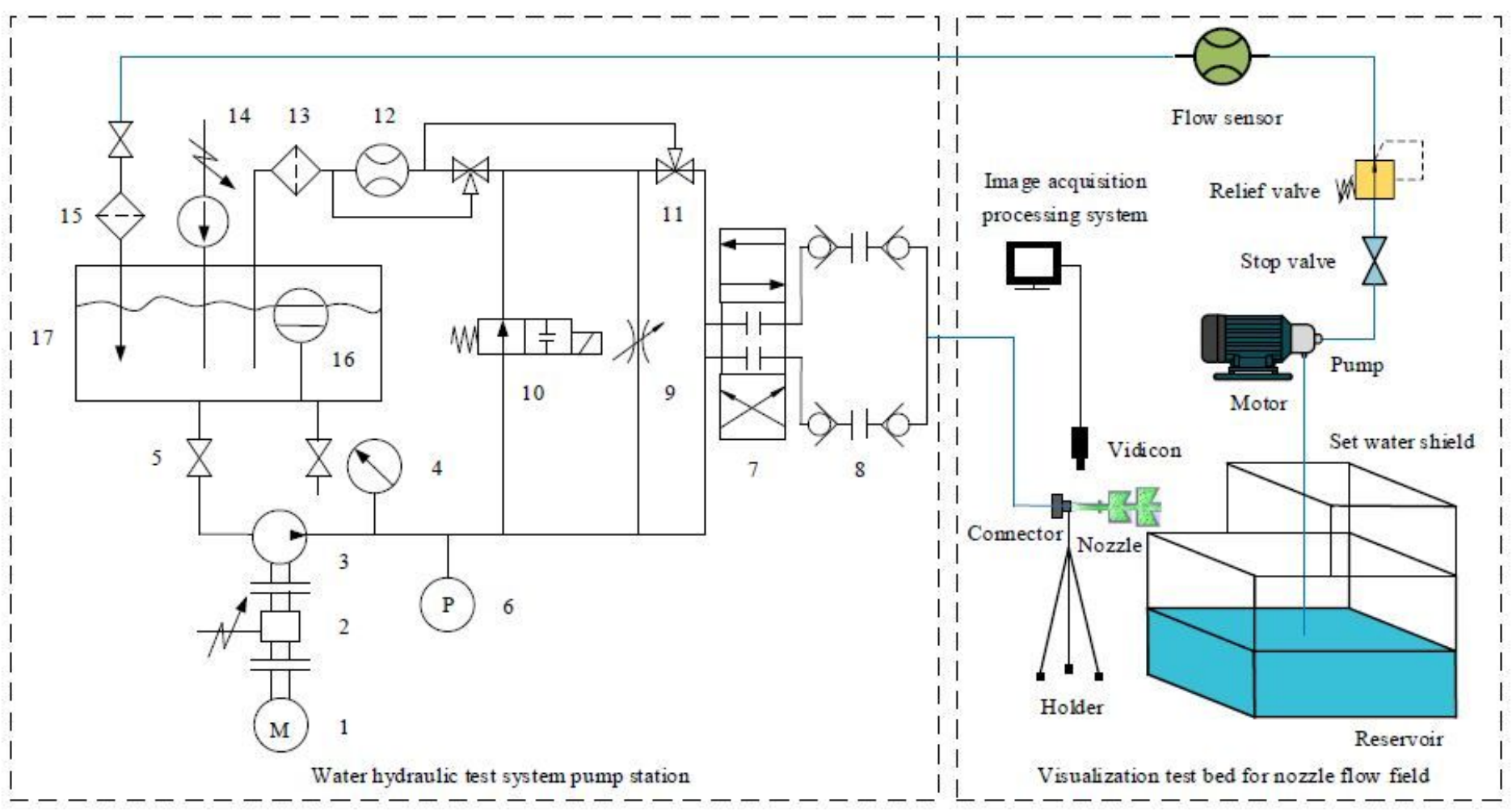

\section{Figure 18}

Visualization Test Device for Flow Field of Self-excited Oscillating Pulse Cavitation Jet Nozzle: 1: motor; 2: Torque tachometer; 3: Water pump; 4: Pressure gage; 5 : Force sensor; 6 : Low pressure cut-off valve; 7 : Three four-way solenoid valve; 8: Quick change connector; 9: Flow control valve; 10: Two position two way valve; 11: Three-way valves; 12: Flow sensor; 13: Graded filter; 14: Temperature sensor; 15: Filling water filter; 16: Liquid level gauge; 17: Water tank

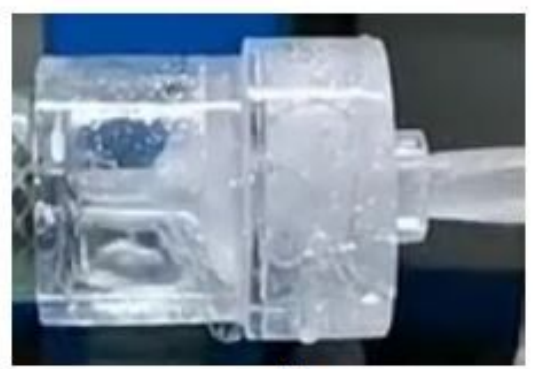

(a)

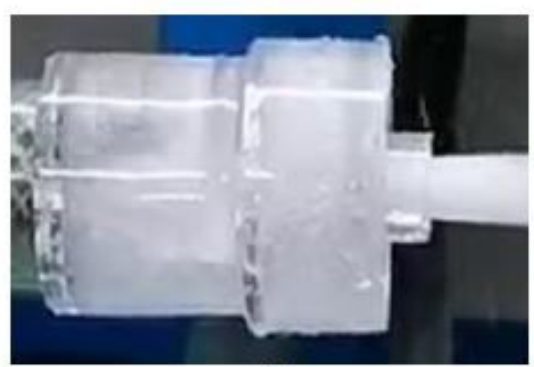

(b)

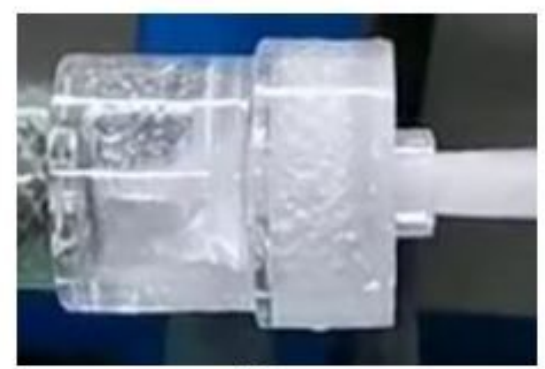

(c)

\section{Figure 19}

Evolution process of flow field of $\mathrm{n}$ ozzle at $0.5 \mathrm{MPa}$ : ( Cavitation development stage; $b$ ) Cavitation aggregation stage $c$ ) Cavitation collapse stage 


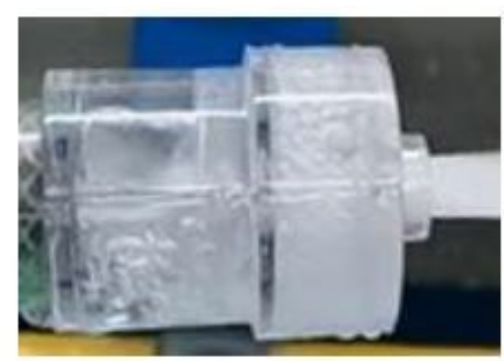

(a)

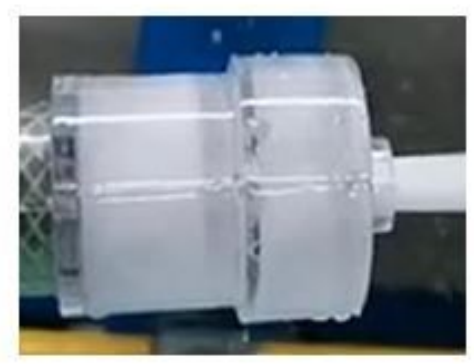

(b)

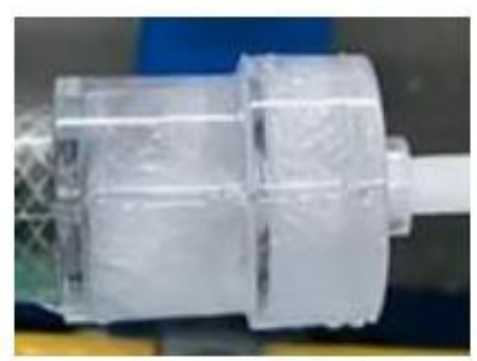

(c)

Figure 20

Evolution process of flow field of nozzle at $3 \mathrm{MPa}$ : ( Cavitation devel opment stage; b ) Cavitation aggregation stage $\mathrm{c}$ ) Cavitation collapse stage

\section{Supplementary Files}

This is a list of supplementary files associated with this preprint. Click to download.

- video1.mp4 